\title{
Integrating Additional Information \\ (Such as H, O, N, S and F) Obtained \\ from the Laser Ablation ICP-MS \\ Process: An Introduction to a Novel \\ Integrated (and Upgradable) LIBS \\ System Within a $193 \mathrm{~nm}$ Laser
}

Ablation System

\author{
ALAN KOENIG AND JHANIS GONZALEZ
}

Applied Spectra, Inc.

Presenting Author: akoenig@appliedspectra.com

Laser ablation ICP-MS (LA-ICP-MS) is well known for powerful trace element and isotopic microanalyses within geosciences. The harnessing of the optical emission spectroscopy emitted during every LA-ICP-MS measurement has been previously demonstrated to add important elements such as $\mathrm{H}, \mathrm{O}$, $\mathrm{N}, \mathrm{S}$ and $\mathrm{F}($ !) and provides complimentary information for the 2D and 3D mapping of geological materials during conventional LA-ICP-MS analyses of elements and isotopes. Here we present a new laser induced breakdown spectroscopy (LIBS) module integrated with (and upgradable to) a $193 \mathrm{~nm}$ LA system.

The fact that this optical emission occurs during all LA analyses means that useful spectroscopic information is wasted in conventional LA systems. This spectroscopic information includes full spectral characterization (fingerprinting) of the mineral. The resulting spectral information can be harvested for powerful multi-variate spectral analyses and this information can reveal subtle sub-populations within a set of grains or spots. The LIBS can also record information for every pulse of the LA analysis, leading to $3 \mathrm{D}$ information, including information about small inclusions (including fluid and gas inclusions) that may be buried within the LA-ICP-MS signal or that may complicate the LA-ICP-MS results.

The figures of merit for analyses of tandem LIBS and LAICP-MS for a variety of important and interesting mineral systems such as apatite, tourmaline and basaltic glass are presented. Leveraging the novel configuration of the $193 \mathrm{~nm}$ LA system, the power of QQQ-ICP-MS (MS/MS) and simultaneous LIBS will add value to high throughput geochronological studies by providing spectral fingerprinting (and correlation) of dated mineral grains as well as access elements such as $\mathrm{F}$ that are not conventionally determined by LA-ICP-MS. A forward look at potential applications and implications of this technique will be presented. 